\title{
Numerical Model of Stepped Leader Growth Process in Homogenous Dielectric
}

\author{
A. Berkopec and V. Valenčič
}

\author{
ZVD Zavod za varstvo pri delu d.d., Ljubljana, Slovenia \\ Faculty of Electrical Engineering, Ljubljana, Slovenia
}

\begin{abstract}
We present a numerical model of stepped leader growth during dielectric breakdown process in homogenous media. The electrodes and the stepped leader were modelled as conductors surrounded by a homogenous dielectric medium. We took in account the observations of a stepped leader in atmosphere and used a quasi-static approach. To simulate the time space, we alternatingly added a streamer to the leader and solved the system for unknown charges with method of moments, using Galerkin approach. For different values of generalized dielectric temperature $k T$ and initial electrical energy $C_{0} U^{2} / 2$ the results of the model were interpreted as various kinds of discharges, like corona, cold discharge, and discharges with branched leaders. The results suggest the existence of a single parameter that determines the type of breakdown in the particular class of discharges.
\end{abstract}

Key words: dielectric breakdown, lightning, method of moments, growth algorihtms

\section{Introduction}

Modelling of growth processes was extensively studied during the past twenty years because of the availability of computer power, for example the simulation of viscous fingers and snowflakes [9] by means of Darcy's law and fixed computational lattice. These models used the weighted random generator for simulation of growth process and produced results that highly resembled the fractal structures of objects in question.

Although the process of charge separation in clouds is still under debate [1], the growth of the stepped leader in lightning is well documented $[3,8]$. On a smaller time scale it resembles the growth of viscous fingers or snowflakes, yet the stepped leader of lightning is not a fractal structure and does not suggest any fixed computational mesh or internal symmetry.

Considering all these facts we designed a computer simulation of stepped leader growth process in which we retained the weighted random process, but dismissed the fixed computational mesh, and computational methods using it, like finite difference 
and finite element methods. The method of moments was most suitable for such a computational task.

\section{Methods}

The model consisted of conductors placed in a homogenous insulator with dielectric constant $\epsilon$. Conductors available in the model were square plates and cylinders with finite radius. Our primary goal was to investigate branching of stepped leader in a simple system of two parallel square plates. The parts of the leader were added with a weighted random generator. The weight was proportional to the electric field strength at a particular point and depended also on the properties of dielectric, initial geometry, and initial electric potentials.

At each step we computed the charge distribution and electric field where necessary. The quasi-static growth of breakdown channel is in accordance with the observations of stepped leader creation process in lightning when a seed of the leader is in negatively charged cloud $[6,3,1]$. The experiments show that stepped leader advances for approximately $50 \mathrm{~m}$ within a microsecond and then waits for about $50 \mu \mathrm{s}$. We assumed that plasma in the stepped leader and charged cloud were on the same electric potential and the ground on the opposite. They were all perfect conductors and there was enough time during $50 \mu \mathrm{s}$ pause for redistribution of charge. Typical relaxation time for charge redistribution in the perfect conductor is of the order $10^{-19} \mathrm{~s}$ [5] which justifies the quasi-static approach.

\subsection{Initial and boundary conditions}

The streamer is started at the middle of upper plate. Initial capacitance of the system is $C_{0}$. There are no additional initial boundary conditions since we assumed the system is in equilibrium at each computational step. As cited above, experiments had shown the growth of the stepped leader has $50 \mu$ s pause after the creation of each additional streamer within $1 \mu \mathrm{s}$. We assume those $50 \mu$ s suffice for electric charges to achieve electrostatic equilibrium.

Parallel plates are on constant potential difference $U$ which does not change during the simulation. The potential of each streamer in stepped leader is the same as that of square plate it started to grow from.

\section{$2.2 \quad$ Electrostatic solver}

The solution of electrostatic potential $V$ satisfies Laplace equation $\nabla^{2} V=0$ [7] and can be obtained from charge distribution $\rho(\mathbf{r})$ as

$$
V(\mathbf{r})=\frac{1}{4 \pi \epsilon} \int_{v^{\prime}} \frac{\rho\left(\mathbf{r}^{\prime}\right)}{\left|\mathbf{r}-\mathbf{r}^{\prime}\right|} d v^{\prime}
$$

where $\mathbf{r}^{\prime}$ is vector on all conductors $v^{\prime}$. The electric potential on conductors satisfied the Dirichlet boundary conditions with charge density locally constant over computational domains $\sigma_{i}=Q_{i} / d A_{i}$ and $q_{i}=Q_{i} / d l_{i}$ (Figure 1 ). 


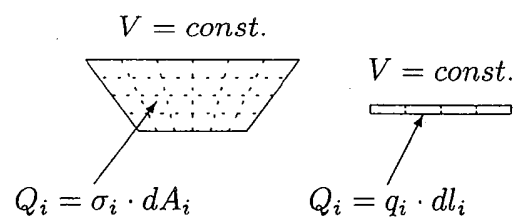

Figure 1: Square plate and line conductor were the basic objects of computational system. In equation $1 d v^{\prime}$ denotes computational domains $d A_{i}$ and $d l_{i}$.

Using Galerkin approach [4] we integrated once more over every computational domain and obtained a linear system of equations for unknown charges $Q_{j}$

$$
\tilde{V}_{i}=\frac{1}{v_{i} v_{j}} \sum_{j} \int_{v_{i}} \int_{v_{j}} \frac{d v_{j} d v_{i}}{\left|\mathbf{r}_{i}-\mathbf{r}_{j}\right|} Q_{j}
$$

where $\tilde{V}=4 \pi \epsilon V$. Defining matrix elements $M_{i j}$ as

$$
M_{i j}=\frac{1}{v_{i} v_{j}} \int_{v_{i}} \int_{v_{j}} \frac{d v_{j} d v_{i}}{\left|\mathbf{r}_{i}-\mathbf{r}_{j}\right|}
$$

we wrote the system of equations as

$$
\left(\begin{array}{ccc}
M_{11} & \ldots & M_{1 n} \\
\vdots & \ddots & \vdots \\
M_{n 1} & \ldots & M_{n n} \\
1 & \ldots & 1
\end{array}\right) \cdot\left(\begin{array}{c}
Q_{1} \\
\vdots \\
Q_{n}
\end{array}\right)=\left(\begin{array}{c}
\tilde{V}_{1} \\
\vdots \\
\tilde{V}_{n} \\
0
\end{array}\right)
$$

We assumed the net charge is conserved, therefore an extra equation $\sum_{j} Q_{j}=0$ was added to the linear system which lead to more equations than unknowns. We solved the system 4 using the singular value decomposition (SVD) algorithm [2].

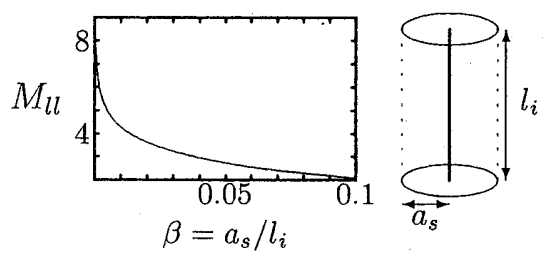

Figure 2: Integral $M_{l l}$ from equation 6 as a function of parameter $\beta=a_{s} / l_{i}$, where $a_{s}$ is radius and $l_{i}$ is length of "line" conductor. Line conductors have a finite radius $a_{s}$, becoming conducting cylinders, since integral 3 is singular for onedimensional objects (cylinders with radius $a_{s}=0$ ).

The diagonal matrix elements are non-singular for 2D objects, like square plate domains. When calculating diagonal elements we assumed line conductors were cylinders 
of finite radius $a_{s}$ and satisfied the boundary condition for $V=$ const. along the axis. This yielded the following equation

$$
M_{l l}=\frac{1}{l_{i}^{2}} \int_{l} \int_{l^{\prime}} \frac{d z d z^{\prime}}{\sqrt{a_{s}^{2}+\left(z-z^{\prime}\right)^{2}}}
$$

with an analytical solution

$$
M_{l l}=\frac{\beta-\sqrt{1+\beta^{2}}-\ln \frac{\sqrt{1+\beta^{2}}-1}{\beta}}{2 / l_{i}} .
$$

All symbols are explained in Fig. 2.

\subsection{Weighted random generator}

The stepped leader was composed of streamers (Figure 3) that were grown one per each calculation of charge distribution. We defined such a structure of the leader that allowed branching of the leader at any end point of any streamer with not more than two attached streamers.

The streamer to be added to the stepped leader structure was chosen among the candidates - the points of the leader, which connected with less than three streamers (the central point in Figure 3 on the right would attribute no candidates since streamers can have no more than two successors). There were typically $K=50$ candidates per each of the $N$ end points with less than three streamers. Coordinates for the candidates were chosen randomly on the sphere of radius $l_{i}, l_{i}$ being the length of each streamer.

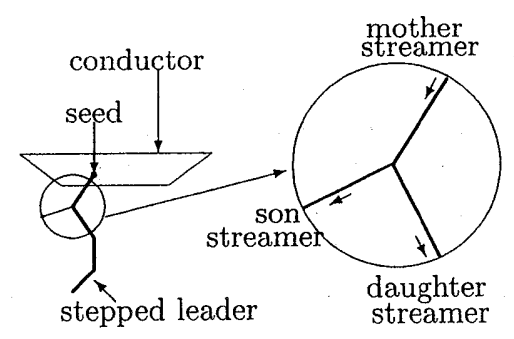

Figure 3: Schematic structure of the stepped leader, composed of streamers. An arbitrary streamer has no successors when created, but can get maximum two successors. The stepped leader grew by one streamer per computational cycle. The particular streamer was chosen with weighted random generator among the candidates.

From the charge distribution the electric potential $V_{n, k}$ of candidate $n, k$ was calculated for each of $N \cdot K$ candidates. It was used to evaluate a weight function $f_{n, k}$ for each of the candidates.

$$
f_{n, k}=\exp \left[\frac{Q_{n} \cdot\left(V_{n, k}-V_{n, k}^{\max }\right)}{k T}\right] .
$$


$V_{n, k}^{\max }$ is maximum value among electric potentials $V_{n, k} . K$ is the number of candidates for each of $N$ points on the leader, points with less then three streamers attached to it. Values of $k$ run form 1 to $K$, and values of $n$ run from 1 to $N$. The streamers are attached to a point as shown on Fig. 3. The point in the middle on Fig. 3 already has three streamers attached to it and is not among $N$ points which produce candidates in the next computational step. Points with less than three streamers attached to them are all among $N$ points, which produce candidates-streamers for continuation of the stepped leader at next step.

The candidate was chosen in the following way: after weight function had been evaluated for all of the $N \cdot K$ candidates, all the values were summed and spanned over the $[0,1)$ interval, keeping track of the interval taken by each candidate. Then random number was picked and the candidate spanning the region of the random number was chosen as a next streamer in the stepped leader.

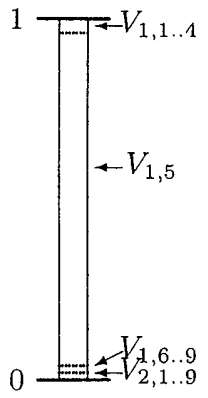

a)

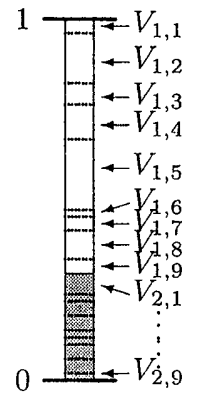

b)

Figure 4: Example of probability function for low (a) and high (b) generalized temperature $k T$.

We chose the particular weight function 7 in accordance with Arrhenius' law of conductivity which approximates the conductivity of the media.

The generalized temperature $k T$ was computed from initial conditions. The quotient of initial electrical energy and generalized temperature of dielectric $k T$ provided a suitable measure of breakdown conditions. With analogy in statistical mechanics we defined a probability for event as

$$
-\ln \left(f_{n, k}\right) \propto \frac{C_{0} U^{2}}{2 k T},
$$

where $C_{0}$ is initial capacitance of the system, $U$ is electric potential, and $C_{0} U^{2} / 2$ is the initial electric energy stored within the system. By defining

$$
p=\frac{C_{0} U^{2}}{2 k T}
$$

the weight function was inversely proportional to temperature $k T$. We describe our results in the parameter $p$ space which is related to weight function $f_{n, k}$ as

$$
f_{n, k} \propto e^{-p}
$$

The parameter $p$ was a part of initial conditions for every simulation. 

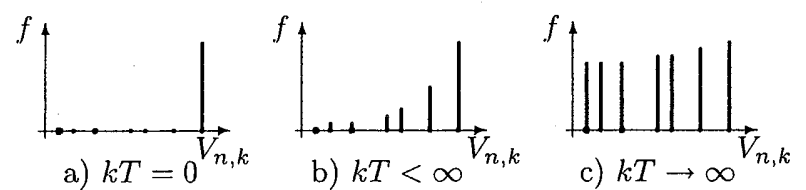

Figure 5: Weight function for different values of $k T$. As $k T$ approaches infinity probabilities for candidates become equal. When $k T=0 \mathrm{~K}$ the weight function filters out the candidate with maximum probability.

\subsection{Algorithm}

The computational algorithm can be summarized in the following steps:

1. preprocessing

- define geometry and electric potentials of conductors

- choose a seed point (usually on one of the conductors, in our case the center point of upper square plate)

- define generalized temperature of dielectric $k T$ (or parameter $p$ )

2. calculating the charge distribution

- compute the matrix elements

- solve the linear system of equations for unknown charges

3. advancing the stepped leader

- define candidates for next streamer

- evaluate the weight function for each candidate

- choose next streamer with weighted random generator

4. final condition

- find minimal distance between leader and conductors of different voltage

- if the distance is less than some $\Delta$ stop, otherwise continue at step 2.

\section{Results}

In order to estimate the computational error several tests were performed with the model of parallel plate condensor and systems of line conductors. One of the test results is shown in Table 1 and Figure 6. It showed the fixed error which is a result of finite computational domains. Since we ran all simulations with typical value of $n=4$ or more, we estimated the fixed error to be below $1 \%$.

The net charge increased with time as shown on Figure 7 for cold and hot discharge. It is not conserved although there is an equation that should have provided such a 

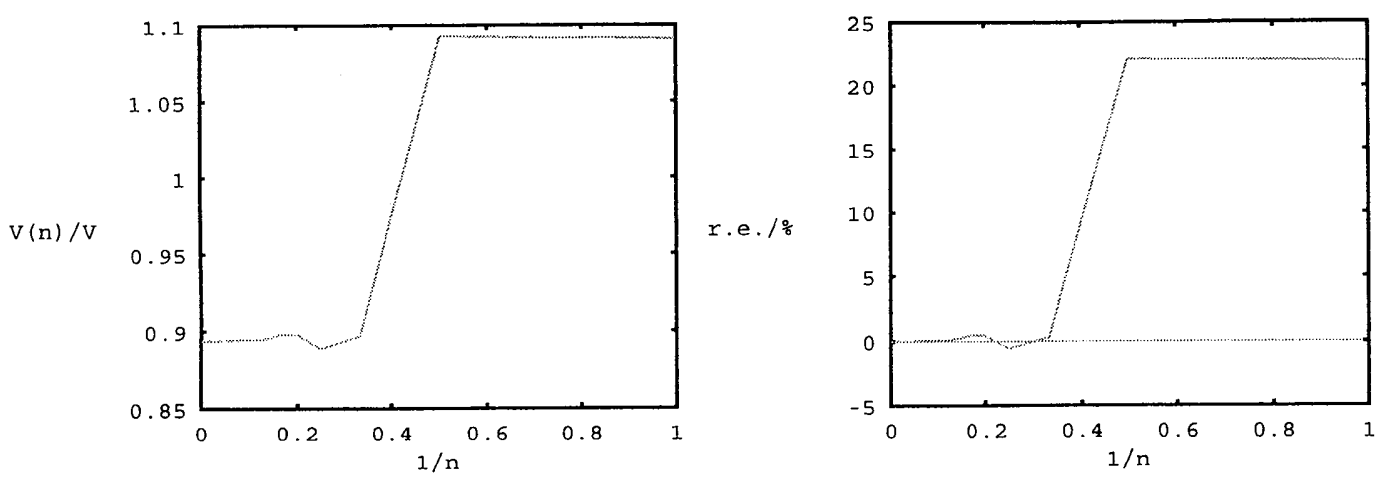

Figure 6: Error estimation as a function of discretization step $n$ in one dimension (square plate has $n \times n$ domains). On the left electric potential, on the right relative error, computed for point $(0.0,0.0,0.45 \mathrm{~m})$ in the system of two parallel charged conducting squares at $z= \pm 0.5 \mathrm{~m}$ with sides along $x$ and $y$, and dimensions $1 \mathrm{~m} \times 1 \mathrm{~m}$.

Table 1: Potential at $(0.0,0.0,0.45 \mathrm{~m})$ for the system of two identical square parallel charged plates at $z= \pm 0.5 \mathrm{~m}$ with sides along $x$ and $y$, and dimensions $1 \mathrm{~m} \times 1 \mathrm{~m}$. $n$ is the number of divisions in one dimension e.g. square plate consists of $n \times n$ domains with constant surface charge density.

\begin{tabular}{|c|c|c|c|}
\hline$n$ & $\Delta=\frac{1}{n}$ & $V(n) / \mathrm{V}$ & $\frac{V(n)-V(\infty)}{V(\infty)} / \%$ \\
\hline 1 & 1.0000 & 1.090674 & 22.02 \\
2 & 0.5000 & 1.092302 & 22.20 \\
3 & 0.3333 & 0.896731 & 0.32 \\
4 & 0.2500 & 0.889044 & -0.54 \\
5 & 0.2000 & 0.898031 & 0.47 \\
6 & 0.1667 & 0.898041 & 0.47 \\
7 & 0.1429 & 0.895711 & 0.21 \\
8 & 0.1250 & 0.894990 & 0.13 \\
$\infty$ & 0.0000 & 0.893861 & 0.00 \\
\hline
\end{tabular}
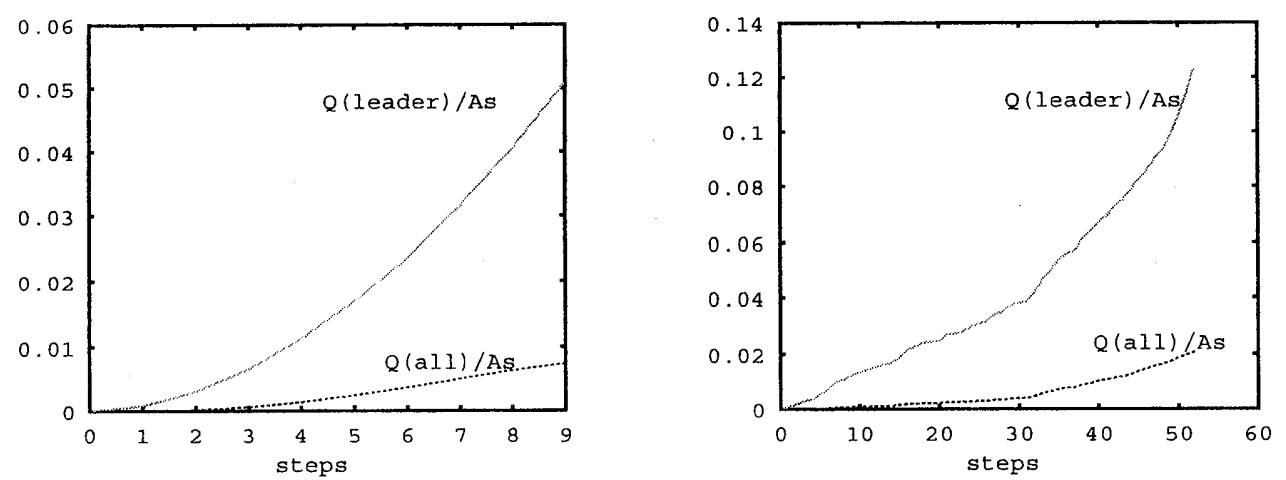

Figure 7: Electric charge $Q$ (leader) on the leader and total electric charge $Q($ all $)$ (measured in Ampereseconds) as a function of number of computational steps for cold dielectric $p>10000$ (left) and hot dielectric $p<100$ (right) for the system of two identical square parallel charged plates at $z= \pm 0.5 \mathrm{~m}$ with sides along $x$ and $y$, and dimensions $1 \mathrm{~m} \times 1 \mathrm{~m}$. Number of computational steps is equal to number of streamers in stepped leader. Electric potentials of plates were $V_{+-}= \pm 1 \mathrm{~V}$. 


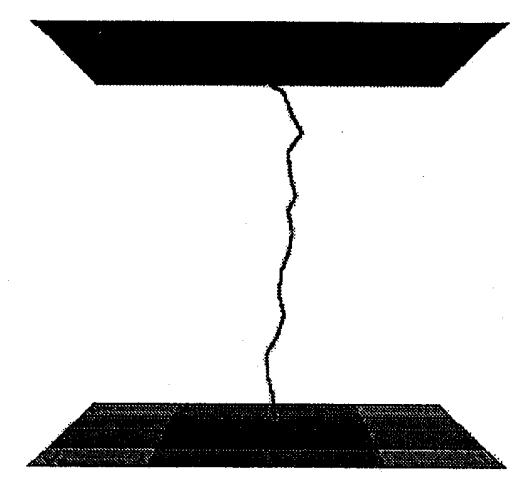

Figure 8: Example of siepped leader for cold discharge with $p=20000$ and $\beta=$ 0.002 . Parameter $\beta$ is a quotient of streamer radius and its length.

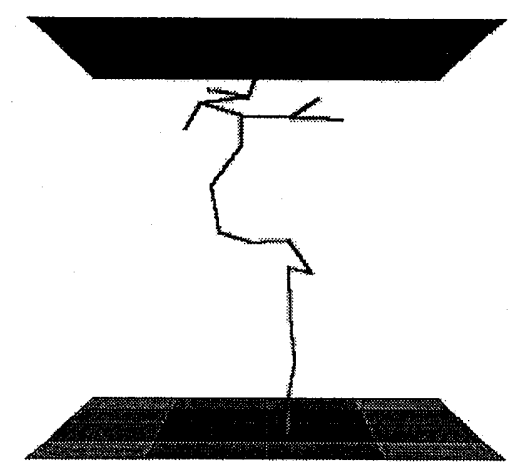

Figure 9: Example of stepped leader with $p=1000$ and $\beta=0.002$. Parameter $\beta$ is a quotient of streamer radius and its length.
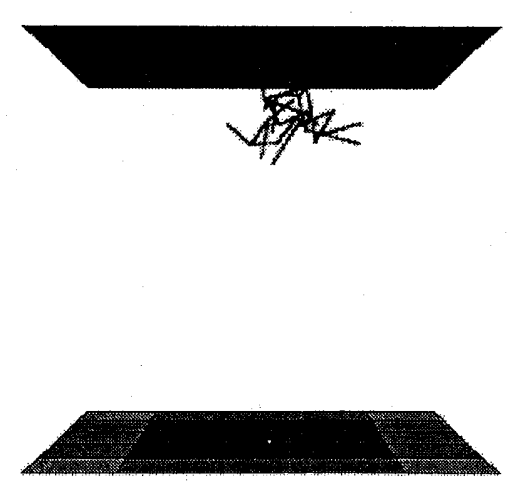

Figure 10: Example of stepped leader for corona discharge with $p=100$ and $\beta=0.002$. Parameter $\beta$ is a quotient of streamer radius and its length. 
solution. This effect is due to the more equations than unknowns in a linear system 4. The solution was best in the least-squares sense.

We made over thousand breakdown simulations for a system of parallel plates for different values of $p$. For typical lightning in Earth atmosphere experiments show approximately $0.1 \mathrm{~m}$ for radius and $50 \mathrm{~m}$ for length of the streamer which lead to $\beta=0.002$. All simulations mentioned or shown (also Figures 8, 9, and 10) in this article were made using value of $\beta=0.002$. Typical results of our computations are shown on Figures 8, 9, and 10.

The $p$-space was divided in two distinctive regions, shown on Figure 11. We interpreted the results of a breakdown path with no branches as "cold discharge" (Figure 8 ) and the one with many branches that even with large number of steps stayed localized around the seed as "corona" (Figure 10). From these observations we limited the cold discharges to values $p \geq 10000$ and the corona to $p \leq 100$. The values of $100 \leq p \leq 10000$ lead to discharges with several branches (Figure 9), the number of branches being inversely proportional to $p$.

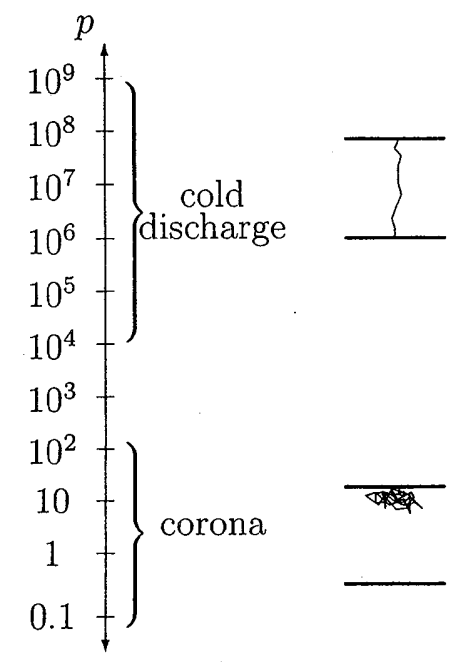

Figure 11: Parameter space of parameter $p$ for fixed value of $\beta=0.002$.

\section{Conclusion}

We built a model with strong connection to a lightning process in a sense that the conductive channel is built successively, like the path of plasma in the stepped leader.

Algorithm alternatingly added a streamer to the leader and calculated the distribution of electric charge on all conductors and the leader as well. The unknown charge distribution was obtained using the method of moments with Galerkin approach. We introduced a generalized temperature of the dielectric $k T$ and defined a parameter $p$ as a measure of electric and dielectric energy. The quotient of both was used as a source for weighted random generator that chose a new streamer for a leader at each step of algorithm. 
The results show that the cold discharge and corona discharge can be obtained from a single computational algorithm with one governing parameter for a fixed value of $\beta$, quotient of diameter and length of a streamer. For specified geometry, the results depend only on quotient of initial electrical energy $C_{0} U^{2} / 2$ and generalized dielectric temperature $k T$.

The interpretation of the stepped leader with branches lacks a firm mathematical description. We considered that the number of branches divided by length of the leader is not a sufficient measure of branching. Therefore we considered mainly two extreme cases: the cold discharge as no branching example and corona as an example with intense branching when leader even after many steps did not reach the conductor of opposite charge. The type of breakdown process for particular geometry was estimated from the quotient of initial electrical energy and the dielectric temperature $k T$ alone. Thermal energy $k T$ of dielectric is very loosely defined, and so is parameter $p$. This is why we believe these results allow more qualitative than quantitative verification.

\section{Acknowledgements}

One of authors (A.B.) would like to thank Institute of Occupational Safety (ZVD Zavod za varstvo pri delu d.d.) from Ljubljana for their computer resources.

\section{References}

[1] R. Feynman, R. Leighton, and M. Sands. The Feynman Lectures on Physics, volume II. California Institute of Technology, 1989.

[2] G. H. Golub and C. F. Van Loan. Matrix Computations. Johns Hopkins University Press, Baltimore, 1996.

[3] C. Magono. Thunderstorms. Elsevier, Amsterdam, 1980.

[4] P. P. Silvester and R. L. Ferrari. Finite Elements for Electrical Engineers. Cambridge University Press, Cambridge, 1986.

[5] A. R. Sinigoj. Fundamentals of Electromagnetic Theory (in Slovene language). Faculty of Electrotehnical Engineering, Ljubljana, 1996.

[6] M. Uman. Lightning. Dover Publications, 1983.

[7] V. Valenčič. Osnove elektrotehnike. Faculty of Electrotechnical Engineering, Ljubljana, 1994.

[8] H. Volland. Atmospheric Electrodynamics. Springer-Verlag, Berlin, 1984.

[9] T. A. Wilson and L. M. Sander. Diffusion limited aggregation. Phys. Rev. B Vol $27,1983$.

(Received October 23, 2000; Revised July 17, 2001; Accepted August 2, 2001) 